\title{
Recognition of Graphs with Convex Quadratic Stability Number
}

\author{
Maria F. Pacheco* and Domingos M. Cardoso ${ }^{\dagger}$ \\ ${ }^{*}$ Department of Mathematics, Polytechnic Institute of Bragança, Portugal \\ ${ }^{\dagger}$ Department of Mathematics, University of Aveiro, Portugal
}

\begin{abstract}
A stable set of a graph is a set of mutually non-adjacent vertices. The determination of a maximum size stable set, which is called maximum stable set, and the determination of its size, which is called stability number, are central combinatorial optimization problems. However, given a nonnegative integer $k$, to determine if a graph $G$ has a stable set of size $k$ is NP-complete. In this paper we deal with graphs for which the stability number can be determined by solving a convex quadratic programming problem. Such graphs were introduced in [13] and are called graphs with convex- $Q P$ stability number. A few algorithmic techniques for the recognition of this type of graphs in particular families are presented.
\end{abstract}

Keywords: graph theory, stability number, combinatorial optimization

PACS: $02.10 .0 \mathrm{x}$

\section{INTRODUCTION}

Graphs with convex- $Q P$ stability number are graphs for which the stability number is equal to the optimal value of a convex quadratic program. It is known that there is an infinite number of graphs with convex- $Q P$ stability number. The purpose of this paper is to present combinatorial characterizations of graphs with convex- $Q P$ stability number and some families of graphs in which it is possible to recognize, in polynomial-time, if a graph has (or not) convex- $Q P$ stability number. Throughout this paper, $G$ will be an undirected simple graph with $n$ vertices for which $V=V(G)$ denotes the nonempty set of the vertices and $E=E(G)$ the set of the edges. We say that vertex $u$ is adjacent to vertex $v$ if they are endpoints of an edge (that will be denoted by $u v$ ) and we call neighborhood of $u$ the set $N_{G}(u)=\{v \in V: u v \in E\}$. Given a vertex $u, d_{G}(u)=\left|N_{G}(u)\right|$ is the degree of $u$. A graph where all vertices have degree $p$ is called a $p$-regular graph. A graph of order $n$ in which all pairs of vertices are adjacent is a complete graph and will be denoted by $K_{n}$. Given a graph $G$ and a set of vertices $U \subseteq V$, the subgraph of $G$ induced by $U$ is graph $G[U]$ such that $V(G[U])=U$ and $E(G[U])=\{u v: u, v \in U$ and $u v \in E(G)\}$. Graph $G$ is strongly regular with parameters $(n, p, a, c)$ if it is a not complete $p$-regular graph, with $p>0$, such that each pair of adjacent vertices has $a$ common neighbors and each pair of non-adjacent vertices has $c$ common neighbors. A graph is bipartite if $V(G)$ is the disjoint union of two sets $V_{1}$ and $V_{2}$ such that $\forall u v \in E(G),\left|V_{1} \cap\{u, v\}\right|=\left|\{u, v\} \cap V_{2}\right|=1$. A set of mutually non adjacent vertices in a graph is called a stable set and a set of mutually adjacent vertices is called a clique. A stable set (clique) $S$ is called maximum stable set (clique) if there is no other stable set (clique) with greater number of vertices. The number of vertices in a maximum stable set (clique) of graph $G$, is called the stability (clique) number of $G$ and is denoted by $\alpha(G)(\omega(G))$. Given graph $G$, the line graph of $G$, which is denoted by $L(G)$, is constructed by taking the edges of $G$ as vertices of $L(G)$ and joining two vertices in $L(G)$ by an edge whenever the corresponding edges in $G$ have a common vertex. A matching in $G$ is a subset of edges, $M \subseteq E(G)$, no two of which have a common vertex. A matching with maximum cardinality is a maximum matching. It is well known that the problem of determining a maximum matching in $G$ is equivalent to the problem of determining a maximum stable set in $L(G)$. Given a subset of vertices $S$ of graph $G$, the vector $x \in \mathbb{R}^{n}$ such that $x_{v}=1$ if $v \in S$ and $x_{v}=0$ if $v \notin S$ is the characteristic vector of $S$. We say that $S \subset V(G)$ is $(\kappa, \tau)$-regular if it induces a $\kappa$-regular subgraph and $\forall u \notin S,\left|N_{G}(u) \cap S\right|=\tau$. Throughout this paper, $A_{G}$ (or $A$ ) will denote the adjacency matrix of $G$, that is $A_{G}=\left(a_{i j}\right)$ will be such that

$$
a_{i j}= \begin{cases}1 & \text { if } i j \in E(G) \\ 0 & \text { if } i j \notin E(G)\end{cases}
$$

and $\lambda_{\max }(A)=\lambda_{1}(A) \geq \lambda_{2}(A) \geq \ldots \geq \lambda_{n}(A)=\lambda_{\min }(A)$ will denote the eigenvalues of $A_{G}$. It is well known that if $G$ has at least one edge, then $\lambda_{\min }\left(A_{G}\right) \leq-1$. Actually, $\lambda_{\min }\left(A_{G}\right)=-1$ if and only if $\mathrm{G}$ has at least one edge and every component of $G$ is complete. 


\section{GRAPHS WITH CONVEX- $Q P$ STABILITY NUMBER}

Let $G$ be a graph of order $n$ with at least one edge and $A_{G}$ its adjacency matrix. Let $v(G)$ denote the optimal value of the quadratic program

$$
v(G)=\max \left\{2 e^{T} x-x^{T}\left(\frac{A_{G}}{-\lambda_{\min }\left(A_{G}\right)}+I_{n}\right) x, x \geq 0\right\}
$$

where $I_{n}$ is the identity matrix of order $n$ and $e$ is the all-one vector.

This program was introduced in [13] in order to obtain an upper bound for the stability number of $G$ and analyzed in [15], where it was proved that its optimal value is the best upper bound for $\alpha(G)$, among a family of convex quadratic programs.

A graph $G$ for which $\alpha(G)=v(G)$ is a graph with convex quadratic stability number or a graph with convex $Q P$ stability number where $Q P$ stands for quadratic programming. The class of graphs with convex quadratic stability number is denoted by $Q$.

It is immediate that program (1) is convex.

In [13], a criteria for equality was given: if $G$ has at least one edge, then $\alpha(G)=v(G)$ if and only if for a maximum stable set $S$ of $G$ (and then for all),

$$
-\lambda_{\min }\left(A_{G}\right) \leq \min \left\{\left|N_{G}(u) \cap S\right|: u \notin S\right\} .
$$
(2).

This condition was improved in [6] where it was proved that $v(G)=\alpha(G)$ if and only if there is a stable set verifying

We should mention that the upper bound $v$ for the stability number of a graph generalizes Hoffman [unpublished] and Lovász's [11] upper bound for the stability number. For regular graphs, we have

$$
v(G)=\frac{-n \lambda_{\min }\left(A_{G}\right)}{\lambda_{\max }\left(A_{G}\right)-\lambda_{\min }\left(A_{G}\right)} .
$$

There are several famous graphs with convex- $Q P$ stability number. It is the case of the Petersen graph which is the strongly regular graph with parameters $(10,3,0,1)$ for which $\lambda_{\min }\left(A_{P}\right)=-2$ and $\alpha(P)=v(P)=4$. It is also the case of the Hoffman-Singleton graph $H S$, the strongly regular graph with parameters $(50,7,0,1)$ for which $\lambda_{\min }\left(A_{H S}\right)=-3$ and $\alpha(H S)=v(H S)=15$ and many others. It is not known if the fourth graph of Moore $M 4$ (that is, the strongly regular graph with parameters $(3250,57,0,1))$ exists. However, if such graph exists then it is expected that it has convex- $Q P$ stability number, with $\lambda_{\min }\left(A_{M 4}\right)=-8$ and $\alpha(M 4)=v(M 4)=400$. Additionally, taking into account [7], graphs defined by the disjoint union of complete subgraphs and complete bipartite graphs are trivial examples of graphs with convex- $Q P$ stability number ( $G$ is a complete bipartite graph if it is bipartite with $V=V_{1} \cup V_{2}$ and $\forall u \in V_{1}, d_{G}(u)=\left|V_{2}\right|=q$ and $\left.\forall v \in V_{2}, d_{G}(v)=\left|V_{1}\right|=p\right)$.

\section{RECOGNITION OF GRAPHS WITH CONVEX- $Q P$ STABILITY NUMBER}

Theorems 3, 4, 5 and 6 in [2] give an algorithmic strategy for the recognition of $Q$-graphs unless the following condition is veryfied:

$$
\forall u \in V, v(G)=v(G-u)=v\left(G-N_{G}(u)\right) \text { and } \lambda_{\min }\left(A_{G}\right)=\lambda_{\min }\left(A_{G-u}\right)=\lambda_{\min }\left(A_{G-N_{G}(u)}\right) .
$$

Graphs having an induced subgraph $G$ with no isolated vertices such that $v(G)$ and $\lambda_{\min }\left(A_{G}\right) \in \mathbb{Z}$, verifying

$$
\forall u \in V, v(G)=v\left(G-N_{G}(u)\right) \text { and } \forall u \in V, \lambda_{\min }\left(A_{G}\right)=\lambda_{\min }\left(A_{G-N_{G}(u)}\right)
$$

are called adverse graphs.

Based in Theorems 3, 4, 5 and 6 in [2], the next procedure recognizes if a graph is (or not) in $Q$ or determines one of its adverse subgraphs. Note that the input of the procedure is graph $G$ and $I s o(H)$ denotes the set of isolated vertices of $H$. 


\section{Procedure 1}

(Input: Graph $\mathrm{G}$ with at least one edge)

1. Set $H:=G, \tau:=-\lambda_{\min }\left(A_{G}\right)$;

2. Set $I=I \operatorname{so}(H), H:=H-I$;

3. If $\exists v \in V(H): \tau \neq-\lambda_{\min }\left(A_{H-N_{H}(v)}\right)$ then

3.1 If $v(G)=v\left(G-N_{G}(v)\right)$ then STOP $(G \in Q)$;

4. If $\exists v \in V(H): v(G-v) \neq v\left(G-N_{G}(v)\right)$ then

4.1 If $v(G) \notin\left\{v(G-v), v\left(G-N_{G}(v)\right)\right\}$ then STOP $(G \notin Q)$ else

4.1.1 If $v(G)=v(G-v)$ then set $G:=G-v$ else set $G:=G-N_{G}(v)$;

4.1.2 gotostep 2 .

else

4.2 If $\exists v \in V(H): v(G) \neq v(G-v)$ then STOP $(G \notin Q)$ else STOP ( $G$ contains the adverse subgraph $H$ );

The following results are useful for the recognition of graphs with convex- $Q P$ stability number:

Theorem 1 If $G$ is adverse, $G \in Q$ if and only if $\exists S \subseteq V(G)$ such that $S$ is $(0, \tau)$-regular with $\tau=-\lambda_{\min }\left(A_{G}\right)$.

Theorem 2 If $G$ is p-regular, $G \in Q$ if and only if $\exists S \subset V(G)$ such that $S$ is $(0, \tau)$-regular with $\tau=-\lambda_{\text {min }}\left(A_{G}\right)$.

As a consequence of Theorem 1 , the recognition of $Q$-graphs can be done applying Procedure 1 or recognizing a $(0, \tau)$-regular set, with $\tau=\lambda_{\min }\left(A_{H}\right)$, in an adverse subgraph $H$ determined aplying the procedure.

\section{ANALYSIS OF PARTICULAR FAMILIES OF GRAPHS}

\section{Bipartite graphs}

According to the Perron-Frobenius Theorem ([10], Theorem 6.1), every connected graph has a simple maximum eigenvalue. On the other hand, when the graph is bipartite, its eigenvalues are symmetric in relation with the origin [9]. Therefore, the minimum eigenvalue of a connected bipartite graph $G$ is simple and then

$$
\exists u \in V(G): \lambda_{\min }\left(A_{G}\right)<\lambda_{\min }\left(A_{G-u}\right) .
$$

Hence, since $G \in Q$ if and only if each component is in $Q$, applying Procedure 1 we can recognize (in polynomial-time) if a bipartite graph is (or not) a $Q$-graph.

\section{Dismantable graphs}

Dismantable graphs have the following recursive definition: the one-vertex graph is dismantable and a graph $G$ with at least two vertices is dismantable if $\exists u, v \in V(G): N_{G}[u] \subseteq N_{G}[v]$ (where $N_{G}[x]=N_{G}(x) \cup\{x\}$ ) and $G-u$ is dismantable. The next Theorem states a neighborhood inclusion condition which does not hold when the graph is adverse and then we may conclude that there are no dismantlable adverse graphs.

Theorem 3 [3] If $G$ has at least one edge and $\exists u, v \in V(G): N_{G}(u) \subseteq N_{G}(v)$, then $v(G)>v\left(G-N_{G}(u)\right)$.

\section{Graphs with low Dilworth number}

Given $u, v \in V(G): N_{G}(u) \subseteq N_{G}(v)$, we say that vertices $u$ and $v$ are comparable. This binary relation is a preorder (that is, it is reflexive and transitive) and is called vicinal preorder. Therefore, graph $D(G)$ such that $V(D(G))=V(G)$ and $E(V(G))=\left\{u, v \in V(G): N_{G}(u) \subseteq N_{G}[v]\right.$ or $\left.N_{G}(v) \subseteq N_{G}[u]\right\}$ is the comparability graph of the vicinal preorder of $G$. Considering the Dilworth number of a graph (its largest number of pairwise incomparable vertices), we have $\operatorname{dilw}(G)=\alpha(D(G))$. 
Theorem 4 [3] Let $G$ be a not complete graph. If $\operatorname{dilw}(G)<\omega(G)$, then $G$ is not adverse.

As a consequence of Theorem 4, we can apply Procedure 1 to recognize in polynomial-time if a graph with Dilworth number equal to 1 (which is a threshold graph) is a $Q$-graph.

\section{Claw-free graphs}

Given a graph $H$, graph $G$ is said to be $H$-free if $G$ has no copy of $H$ as an induced subgraph. A claw-free graph is a $K_{1,3}$-free graph. Also, an $\alpha$-redundant subset of vertices is a subset $U \subseteq V(G): \alpha(G)=\alpha(G-U)$.

The next Theorem allows the recognition of $\alpha$-redundant subsets of vertices in claw-free graphs with comparable non-adjacent vertices, in the sense of the vicinal pre-order relation already defined.

Theorem 5 Let $G$ be a claw-free graph and $u, v \in V(G): u v \notin E(G)$. If $N_{G}(u) \subseteq N_{G}(v)$, then $N_{G}(u)$ is an $\alpha$-redundant subset of vertices.

\section{CONCLUSIONS}

It is expected that, additionally to the referred cases, there are many other classes of graphs in which we may recognize in polynomial-time if a graph has or not convex- $Q P$ stability number. The determination of such classes remains open.

Some other future challenges are the recognition of $\left(0,-\lambda_{\min }\left(A_{G}\right)\right)$ - regular sets in adverse graphs and the recognition in polynomial time of adverse graphs, so that the recognition of graphs with convex- $Q P$ stability number can be made based upon the results here presented in the following way:

1. Aplying Procedure 1 to determine if graph $G$ belongs to the class $Q$ or to determine an adverse subgraph of $G$;

2. Recognizing if the adverse subgraph determined with the procedure has a $\left(0,-\lambda_{\min }\left(A_{G}\right)\right)$-regular set.

\section{REFERENCES}

1. R. Barbosa and D. Cardoso, On regular-stable graphs, Ars Combinatoria, 70: 149-159, (2004).

2. D. Cardoso, Convex quadratic programming approach to the maximum matching problem, Journal of Global Optimization, 21: 91-106, (2001).

3. D. Cardoso, On graphs with stability number equal to the optimal value of a convex quadratic program, Matemática Contemporânea - a publication of the Brazilian Mathematica Society, (2003).

4. D. Cardoso, C. Delorme and P. Rama Laplacian eigenvectors and eigenvalues and almost equitable partitions, European Journal of Combinatorics, 28 : 665-673, (2007).

5. D. Cardoso and C. Luz Extensions of the Motzkin-Straus result on the stability number of graphs, Cadernos de Matemática da Universidade de Aveiro, (2001).

6. D. Cardoso and D. Cvetković Graphs with least eigenvalue -2 attaining a convex quadratic upper bound for the stability number, Cadernos de Matemática da Universidade de Aveiro, (2006).

7. D. Cardoso and P. Rama Equitable bipartitions of graphs and related results, Journal of Mathematical Sciences, 120: 869-880, (2004).

8. D. Cardoso and P. Rama Spectral results on regular graphs with $(\kappa, \tau)$-regular sets, Discrete Mathematics, 307: 1306-1316, (2007).

9. D. Cvetkovic, M. Doob and H. Sachs Spectra of graphs, Academic Press, New York, (1979).

10. C. D. Godsil, Algebraic Combinatorics, Chapman and Hall, New York, (1993).

11. L. Lovász, On the shannon capacity of a graph, IEEE Transactions on Information Theory, $25: 1-7,(1979)$.

12. L. Lovász An algorithmic theory of numbers, graphs and convexity, Regional Conference Series in Applied Mathematics, SIAM, Philadelphia, (1986).

13. C. Luz An upper bound on the independence number of a graph computable in polynomial time, Operations Research Letters, 18: 139-145, (1995).

14. C. Luz, Relating the Lovasz theta number with some convex quadratic bounds on the stability number of a graph, Departamento de Matemática da Universidade de Aveiro, Cadernos de Matemática, (2003)

15. C. Luz and D. Cardoso A generalization of the Hoffman-Lovász bound on the independence number of a graph, Annals of Operations Research , 81: 307-319, (1998).

16. C. Luz and A. Schrijver A convex quadratic characterization of the Lovász theta number, Discrete Math., 19: 382-387, (2005). 